\title{
A relation between Lyapunov exponents, Hausdorff dimension and entropy
}

\author{
ANTHONY MANNING \\ Mathematics Institute, University of Warwick, Coventry CV4 7AL, England
}

(Received 24 July 1981)

Abstract. For an Axiom A diffeomorphism of a surface with an ergodic invariant measure we prove that the entropy is the product of the positive Lyapunov exponent and the Hausdorff dimension of the set of generic points in an unstable manifold.

Recent work in dynamical systems and ergodic theory shows that a diffeomorphism satisfying some hyperbolicity conditions, such as those of Anosov, has very many ergodic invariant measures. These can arise as equilibrium states [21], [3] for real-valued functions and they are singular with respect to each other. We consider a diffeomorphism $f$ of a surface and investigate the disjoint sets $G_{\mu}$ of future generic points [6] on which such measures $\mu$ are concentrated. The Hausdorff dimension $\delta_{\mu}$ of the intersection of $G_{\mu}$ with an unstable manifold is an indication of how thick $\mu$ is. Each measure $\mu$ determines a positive Lyapunov exponent $\chi_{\mu}$ that measures the exponential rate of growth of $\left\|D f_{x}^{n} v\right\|$ for almost all vectors $v$ at $\mu$-almost all points $x$. Our main result is that the entropy $h_{\mu}$ is equal to the product $\delta_{\mu} \chi_{\mu}$. Further ideas on this subject follow the proof.

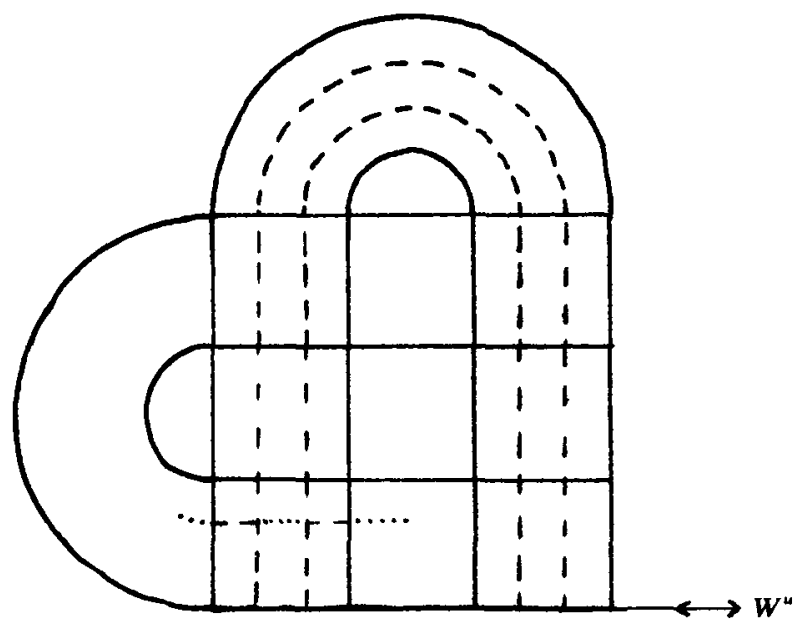

Figure 1 
Example. The horseshoe diffeomorphism [22], as shown in figure 1, can be defined to expand one side of its rectangles by a factor of 3 and contract the other by $\frac{1}{3}$ and in this way to leave non-wandering a middle third Cantor set in each local unstable manifold. The measure $\mu$ of maximal entropy has

$$
h_{\mu}=\log 2
$$

and $\mu$ is evenly distributed on the Cantor set giving

$$
\delta_{\mu}=\log 2 / \log 3 .
$$

Moreover, the Lyapunov exponent is $\log 3$ for any measure. In this simple case our formula reads

$$
h_{\mu}=\log 2=(\log 2 / \log 3) \log 3=\delta_{\mu} \chi_{\mu} .
$$

THEOREM. Let $f: M \rightarrow M$ be a $C^{1}$ Axiom A diffeomorphism of a surface $M^{2}$ preserving an ergodic Borel probability measure $\mu$. Define $G_{\mu}$ to be the set of future generic points for $\mu$. Let $\Omega_{1}$ be the basic set of f for which $G_{\mu} \subset W^{s}\left(\Omega_{1}\right)$. Then the Hausdorff dimension, denoted by $\delta_{\mu}$, of $G_{\mu} \cap W_{\mathrm{loc}}^{u}(x)$ is independent of $x \in \Omega_{1}$. If $\chi_{\mu}$ denotes the positive Lyapunov exponent corresponding to $\mu$ then the entropy $h_{\mu}$ of $f$ is given by the formula

$$
h_{\mu}=\delta_{\mu} \chi_{\mu}
$$

As the proof of our theorem will depend on Bowen's definition in [2] of a topological entropy $h(f, Y)$ for a possibly non-compact subset $Y$ of a compact space $X$ and a continuous map $f: X \rightarrow X$, we recall his definition now. Let $\mathscr{A}$ be a finite open cover of $X$ and write $E<\mathscr{A}$ if $E$ is contained in some member of $\mathscr{A}$. Write $n_{\mathscr{A}}(E)$, or simply $n(E)$, for the largest non-negative integer such that

$$
f^{k} E<\mathscr{A} \text { for } 0 \leq k<n_{\mathscr{A}}(E) \text {. }
$$

If $\mathscr{E}=\left\{E_{1}, E_{2}, \ldots\right\}$ has union containing $Y$, set

$$
D_{\mathscr{A}}(\mathscr{E}, \lambda)=\sum_{i=1}^{\infty} \exp \left(-\lambda n_{\mathscr{A}}\left(E_{i}\right)\right)
$$

Define $m_{\mathscr{A}, \lambda}$ by

$$
\begin{aligned}
& m_{\mathscr{A} A \lambda}(Y)=\lim _{\varepsilon \rightarrow 0} \inf \left\{D_{\mathscr{A}}(\mathscr{E}, \lambda) ;\right. \\
& \left.\mathscr{E}=\left\{E_{1}, E_{2}, \ldots\right\}, \bigcup_{i=1}^{\infty} E_{i} \supset Y \text { and } \exp -n_{\mathscr{A}}\left(E_{i}\right)<\varepsilon \text { for each } i\right\} .
\end{aligned}
$$

Then define

$$
h_{\mathscr{A}}(f, Y)=\inf \left\{\lambda ; m_{\mathscr{A}, \lambda}(Y)=0\right\}
$$

and

$$
h(f, Y)=\sup _{\mathscr{A}} h_{\mathscr{A}}(f, Y) .
$$

Thus $h(f, Y)$ is defined in a way that resembles Hausdorff dimension but with the diameter of a set replaced by the length of time for which its images remain finer than a given cover. 
Hausdorff dimension $\delta(Y)$ is defined, for example in [11], by

$$
\delta(Y)=\inf \left\{\lambda ; m_{\lambda}(Y)=0\right\},
$$

where

$$
m_{\lambda}(Y)=\lim _{\varepsilon \rightarrow 0} \inf \left\{\sum_{i=1}^{\infty}\left(\operatorname{diam} E_{i}\right)^{\lambda} ; \bigcup_{i=1}^{\infty} E_{i} \supset Y \text { and diam } E_{i}<\varepsilon \text { for each } i\right\} .
$$

Proof of theorem. Note first that, if $\Omega_{1}$ has a splitting $T_{\Omega_{1}} M=E^{s} \oplus E^{u}$ with $E^{u}$ or $E^{s}$ two-dimensional, then $\mu$ is concentrated on a periodic orbit and both $h_{\mu}$ and $\delta_{\mu}$ are zero. Thus we need only consider the case of a basic set $\Omega_{1}$ where $E^{s}$ and $E^{u}$ are one-dimensional. If one point $y$ is forward generic for $\mu$ (i.e. the time average along the orbit of $y$ of any continuous function $\psi$ converges to $\mu(\psi)$ ) then so is any other point in the same stable manifold. Thus $G_{\mu}$ is a union of stable manifolds and we cut this set by a special one-dimensional transversal $W_{\text {loc }}^{u}(x)$. As in [3], define a continuous function $\phi^{(u)}: W^{u}\left(\Omega_{1}\right) \rightarrow \mathbb{R}$ by

$$
\phi^{(u)}(y)=-\log \left\|D f_{y} \mid T_{y} W^{u}(y)\right\| .
$$

Then

$$
\chi_{\mu}=-\mu\left(\phi^{(u)}\right)
$$

and, by taking a Riemannian metric adapted to $f[15$, p. 162], we can assume that $\phi^{(u)}$ takes only negative values.

In [2] Bowen showed that $h_{\mu}=h\left(f, G_{\mu}\right)$. We shall start from this and prove that

$$
h\left(f, G_{\mu}\right)=\delta_{\mu} \chi_{\mu} .
$$

Denote $h_{\mu}, \delta_{\mu}, \chi_{\mu}$ simply by $h, \delta, \chi$. The method is for any $\varepsilon>0$ first to prove that, since $G_{\mu}$ has a cover with $D_{\mathscr{A}}(\cdot, h+\varepsilon)$ small, intersecting with $W_{\text {loc }}^{u}(x)$ gives a cover with small $m_{(h+\varepsilon) /(x-2 \varepsilon)}$ and so

$$
\delta \leq(h+\varepsilon) /(\chi-2 \varepsilon) .
$$

Secondly, we shall prove that, since $G_{\mu} \cap W_{\text {loc }}^{u}(x)$ has zero Hausdorff measure in dimension $\delta+\varepsilon$, an economical cover in this sense provides an economical cover for $G_{\mu}$ with small $D_{\mathscr{A}}(\cdot,(\delta+\varepsilon)(\chi+2 \varepsilon))$ so that

$$
h \leq(\delta+\varepsilon)(\chi+2 \varepsilon) \text {. }
$$

We shall use the restriction of the Riemannian metric to the $C^{1}$ embedded compact interval $W_{\text {loc }}^{u}(x)$. Since this is Lipschitz equivalent to the metric as a subset of $M$, it gives the same value of Hausdorff dimension.

Step 1. Given $\varepsilon>0$ choose an open cover $\mathscr{A}$ of $\Omega_{1}$ by rectangles small enough so that $\phi^{(u)}$, which is continuous on the compact set $W_{\varepsilon^{\prime}}^{u}\left(\Omega_{1}\right)$, varies by at most $\varepsilon$ on the region enclosed by each rectangle. Here

$$
W_{\varepsilon^{\prime}}^{u}\left(\Omega_{1}\right)=\bigcup_{x \in \Omega_{1}} W^{u}\left(x, \varepsilon^{\prime}\right),
$$

where $W^{u}\left(x, \varepsilon^{\prime}\right)=\left\{y \in M ; d\left(f^{j} y, f^{j} x\right) \leq \varepsilon^{\prime}\right.$ for $\left.j \leq 0\right\}$. Then for any $\alpha>0$ there is a finite open cover $\mathcal{U}$ of $G_{\mu}$ with

$$
D_{\mathscr{A}}(\mathcal{U}, h+\varepsilon)=\sum_{U \in \mathcal{U}} \exp \left(-n_{\mathscr{A}}(U)(h+\varepsilon)\right)<\alpha .
$$


Let $W$ be any closed interval on any $W^{u}(x)$, so $W$ is compact. Provided $\varepsilon$ is small, $\mathscr{A}$ is so fine that $W$ crosses each $A \in \mathscr{A}$ at most once. Define

$$
G_{\mu, r}=\left\{x \in G_{\mu} ;\left|m^{-1} \sum_{i=0}^{m-1} \phi^{(u)}\left(f^{i} x\right)+\chi\right| \leq \varepsilon, \forall m \geq r\right\}
$$

and note that $G_{\mu}=\bigcup_{r=0}^{\infty} G_{\mu, r}$, since $\mu$ is ergodic and $\chi=-\mu\left(\phi^{(u)}\right)$. In the special case of an algebraic Anosov diffeomorphism of $T^{2}$ we have $\phi^{(u)}$ constant and $G_{\mu}=G_{\mu, 0}$.

Fix $r$. Provided $\alpha$ is small enough we have $n(U) \geq r$ for each $U$ in $\mathscr{U}$. The mean value theorem gives

$$
\begin{aligned}
\operatorname{diam}\left(W \cap G_{\mu, r} \cap U\right) & =\operatorname{diam}^{n(U)}\left(W \cap G_{\mu, r} \cap U\right) /\left\|D f_{y}^{n(U)} \mid E_{y}^{u}\right\| \\
& \leq \operatorname{mesh}_{u} \mathscr{A} /\left\|D f_{y}^{n(U)} \mid E_{y}^{u}\right\|
\end{aligned}
$$

for some $y$ in the convex hull in $W$ of $W \cap G_{\mu, r} \cap U$. Here $\operatorname{mesh}_{u} \not{A}$ denotes the longest interval of an unstable manifold in an element of $\mathscr{A}$. Since the orbit of $y$ from time 0 to $n$ keeps in the same element of $\mathscr{A}$ as that of some point of $G_{\mu, r}$ we have

$$
\left\|D f_{y}^{n(U)} \mid E_{y}^{u}\right\| \geq \exp [(\chi-2 \varepsilon) n(U)] .
$$

Thus the cover

$$
\mathcal{U}^{\prime}=\left\{W \cap G_{\mu, r} \cap U ; U \in \mathscr{U}\right\}
$$

has

$$
\begin{aligned}
\sum_{U^{\prime} \in \mathscr{U}^{\prime}}\left(\operatorname{diam} U^{\prime}\right)^{(h+\varepsilon) /(x-2 \varepsilon)} & \leq\left(\operatorname{mesh}_{\mathcal{u}} \mathscr{A}\right)^{(h+\varepsilon) /(x-2 \varepsilon)} \sum_{U \in \mathscr{U}} \exp [-(h+\varepsilon) n(U)] \\
& <\alpha\left(\operatorname{mesh}_{\mathcal{u}} \mathscr{A}\right)^{(h+\varepsilon) /(\chi-2 \varepsilon)} .
\end{aligned}
$$

Now $\mathcal{U}^{\prime}$ can be made as fine as required by making $\mathcal{U}$ fine. Thus the Hausdorff dimension of $W \cap G_{\mu, r}$ is at most $(h+\varepsilon) /(\chi-2 \varepsilon)$. By taking a countable union over $r$ and then letting $\varepsilon \rightarrow 0$ we obtain

$$
\delta_{W} \leq h / \chi
$$

as required for step 1 , where $\delta_{W}$ is the Hausdorff dimension of $W \cap G_{\mu}$.

Step 2. Given $\varepsilon>0$, again let $\mathscr{A}$ be a covering of $\Omega_{1}$ by open rectangles on each of which $\phi^{(u)}$ varies by at most $\varepsilon$. Let $l$ be a Lebesgue number for $\mathscr{A}$ with the property that any subset of $\Omega_{1}$ is contained in a set in $\mathscr{A}$ if it is small enough for the diameter of its intersection with any local stable or unstable manifold to be less than $l$. Now take any interval $W$ in any unstable manifold and choose $m$ so large that

$$
f^{m} W \cap W^{s}\left(x, \frac{1}{2} l\right) \neq \varnothing \quad \text { for every } x \in \Omega_{1} .
$$

For each $r$ the Hausdorff dimension of $f^{m} W \cap G_{\mu, r}$ is at most $\delta=\delta_{W}$, so we can take a very fine cover $\mathcal{U}_{r}$ of $f^{m} W \cap G_{\mu, r}$ by open intervals in $f^{m} W$ satisfying

$$
\sum_{U \in \mathscr{U}_{r}}(\operatorname{diam} U)^{\delta+\varepsilon}<2^{-r} \text {. }
$$

For each $U \in \mathcal{U}_{\mathrm{r}}$ define $U^{*}$ as $\bigcup_{x \in U \cap \Omega} W^{s}\left(x, \frac{1}{2} l\right)$. Then $f^{n} U^{*}$ is contained in some element of $\mathscr{A}$ so long as $\operatorname{diam} f^{n} U<l$. The ratio of $\operatorname{diam} f^{n} U$ to $\operatorname{diam} U$ is $\left\|D f_{y}^{n} \mid E_{y}^{u}\right\|$ 
for some $y \in U$ and, since the orbit of $y$ from time 0 to $n\left(U^{*}\right)$ is close to that of a point in $U \cap G_{\mu, r}$ and $U_{r}$ can be chosen fine enough so that $n\left(U^{*}\right)>r$, we have

$$
\left\|D f_{y}^{n\left(U^{*}\right)} \mid E_{y}^{u}\right\| \leq \exp \left[(\chi+2 \varepsilon) n\left(U^{*}\right)\right] .
$$

Thus $n\left(U^{*}\right)$ satisfies

$$
l \leq \operatorname{diam} U \cdot \exp \left[(\chi+2 \varepsilon) n\left(U^{*}\right)\right]
$$

and so

$$
\begin{aligned}
\sum \exp \left[-(\delta+\varepsilon)(\chi+2 \varepsilon) n\left(U^{*}\right)\right] & \leq l^{-(\delta+\varepsilon)} \sum(\operatorname{diam} U)^{\delta+\varepsilon} \\
& <l^{-(\delta+\varepsilon)} 2^{-r} .
\end{aligned}
$$

Every point of $G_{\mu} \cap \Omega_{1}$ is in $W^{s}\left(x, \frac{1}{2} l\right)$ for some $x \in f^{m} W \cap G_{\mu, r}$ for all sufficiently large $r$. By combining all $U^{*}$ obtained from the covers $\mathscr{U}_{r}$ for all $r>q$ we obtain a cover $U_{a}^{*}$ of $G_{\mu} \cap \Omega_{1}$ with

$$
\sum_{U^{*} \in \mathfrak{U}_{a}^{*}} \exp \left[-(\delta+\varepsilon)(\chi+2 \varepsilon) n\left(U^{*}\right)\right] \leq l^{-(\delta+\varepsilon)} 2^{-q} .
$$

Thus

$$
h_{\mathscr{A}} \leq(\delta+\varepsilon)(\chi+2 \varepsilon) .
$$

For open covers $h_{\mathscr{A}} \geq h_{\mathscr{B}}$ when $\mathscr{A}$ refines $\mathscr{B}$, so that $h=\sup _{\mathscr{A}} h_{\mathscr{A}}$ is actually a limit of $h_{s A}$ as the mesh of $\mathscr{A}$ tends to 0 . Thus

$$
h \leq\left(\delta_{W}+\varepsilon\right)(\chi+2 \varepsilon) .
$$

Now $\varepsilon$ was arbitrary so

$$
h \leq \delta_{w \chi}
$$

as required for step 2 . Hence

$$
h=\delta_{W \chi}
$$

for any $W$ and the theorem is proved.

Remarks. It is interesting to compare our formula

$$
h_{\mu}=\delta_{\mu} \chi_{\mu}
$$

with Pesin's formula. Pesin proves in [17], for a smooth measure $\mu$ preserved by a general $C^{2}$ diffeomorphism, that $h_{\mu}$ is the integral with respect to $\mu$ of $\chi^{+}$, the sum of the positive Lyapunov exponents. In our two-dimensional case there is only one positive exponent so an ergodic invariant smooth measure has $h_{\mu}=\chi_{\mu}$. Pesin needs the $C^{2}$ (or $C^{1+\alpha}$ ) hypothesis to achieve absolute continuity of the foliations by stable and unstable manifolds. A $C^{2}$ Anosov diffeomorphism of a twodimensional manifold has foliations that are actually $C^{1}$, see [10, theorem 6.3]. In this case a smooth measure has $\delta_{\mu}=1$, so that our theorem gives $h_{\mu}=\chi_{\mu}$ in agreement with Pesin.

Pesin's $C^{2}$ hypothesis is only needed to prove that

$$
h_{\mu} \geq \mu\left(\chi^{+}\right) \text {. }
$$

The opposite inequality

$$
h_{\mu} \leq \mu\left(\chi^{+}\right)
$$




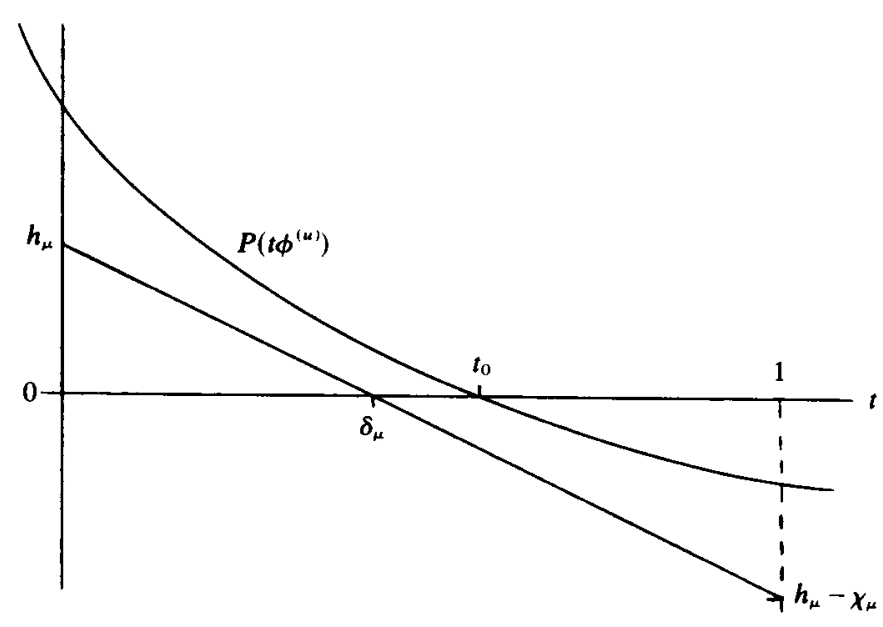

Figure 2

is due to Margulis and only requires $f$ to be $C^{1}$, see $[17, \S 1.6]$. A proof of this by Ruelle for a general measure $\mu$ is in [19]. Indeed $h_{\mu}<\mu\left(\chi^{+}\right)$holds for Axiom A systems if $P\left(\phi^{(u)}\right)<0$ and hence for $C^{2}$ basic sets other than attractors [3]. Our theorem shows for surfaces why the inequality can be strict. (Compare the discussion of this question in [12, p. 140].) It is because a one-dimensional unstable manifold can meet a basic set, that is not an attractor, in a set of Hausdorff dimension $t_{0}$ less than 1 and $\delta_{\mu}$ has at most this value. McCluskey and Manning have shown in [14] that $t_{0}$ is the unique value of $t$ satisfying

$$
P\left(t \phi^{(u)}\right)=0 .
$$

Thus the convex curve $P\left(t \phi^{(u)}\right)$ crosses the axis at $t_{0}$, while the straight line $h_{\mu}-t_{\chi_{\mu}}$ crosses at $\delta_{\mu}$. The straight line can never be above the pressure curve so

$$
\delta_{\mu} \leq t_{0}
$$

Moreover, $\delta_{\mu}=t_{0}$ only holds if $\mu$ is an equilibrium state for $t_{0} \phi^{(u)}$. By the existence and uniqueness of equilibrium states, a $C^{2}$ Axiom $\mathrm{A}$ diffeomorphism of a surface has on each basic set a unique ergodic invariant probability measure $\bar{\mu}$ whose set of generic points in an unstable manifold has maximal Hausdorff dimension. The first part of the proof of lemma 10 in [4] now shows that the set of generic points of $\bar{\mu}$ in an unstable manifold has full Hausdorff $t_{0}$-measure in the set of points of the basic set in this unstable manifold. Thus $\bar{\mu}$ may be considered the Bowen-Ruelle measure for the basic set generalizing the measure defined by Bowen-Ruelle in [5] for $C^{2}$ attractors.

In the $C^{1}$ case $\mu\left(\phi^{(\mu)}\right)=\chi_{\mu}$ depends continuously on the measure $\mu$ in the weak topology and, since $f \mid \Omega$ is expansive, $h_{\mu}$ depends upper semicontinuously on $\mu$ by (16.7) of [6]. It now follows from the theorem that $\delta_{\mu}$ depends upper semicontinuously on $\mu$.

Our calculations do not work for diffeomorphisms of manifolds of dimension greater than two if the measure $\mu$ has more than one positive exponent, $\chi_{1}, \ldots, \chi_{r}$, 
say. We might guess that the set of generic points is aligned with the directions corresponding to the exponents and has a Hausdorff dimension $\delta_{1}, \ldots, \delta_{\text {r }}$ with $0 \leq \delta_{i} \leq 1$ in these directions and that

$$
h_{\mu}=\sum_{1}^{r} \delta_{i} \chi_{i}
$$

A first step would be to extend our theorem to non-uniformly hyperbolic diffeomorphisms of surfaces.

However, when working in a shift space, we can take a metric in which all positive exponents are the same and all expansion seems to take place in the same direction, even though there is no manifold structure in which directions or exponents can be defined. Let $\sigma: \Sigma_{N} \rightarrow \Sigma_{N}$ denote the full one-sided shift on $N$ symbols. Given $a>1$ we define a metric on $\Sigma_{N}$ by $d(x, y)=a^{-k}$, where $k$ is the largest non-negative integer for which $x_{i}=y_{i}$ for all $0 \leq i<k$. Then $\Sigma_{N}$ has diameter 1 and points are moved apart by $\sigma$ with exponent $\log a$. The calculations in the proof of the theorem with $\mathscr{A}$ the cover by cylinders of a certain length and $\chi=\log a$ independent of $\mu$ now prove the following: any ergodic $\sigma$-invariant probability measure $\mu$ on $\Sigma_{N}$ (with support not necessarily all of $\Sigma_{N}$ ) has the Hausdorff dimension $\delta_{\mu}$ of its set of generic points given by

$$
h_{\mu}(\sigma)=\delta_{\mu} \log a
$$

In the case $a=N$ this reads

$$
h_{\mu}(\sigma)=\delta_{\mu} \log N=\delta_{\mu} h_{\text {top }}(\sigma)
$$

so that $\delta_{\mu} \leq 1$. (This corresponds to the fact that $z \rightarrow z^{N}$ on the circle, whose dimension is 1 , is a Lipschitz image of the $N$-shift by a map that preserves the diameter of cylinders in this metric. See [9] and [2, p. 127].) Adler and Marcus have recently used topological entropy to classify subshifts of finite type up to 'almost topological conjugacy', a notion involving factor maps that are one-one except on some universally null set such as the set of non-doubly transitive points [1]. This set is, as they say, negligible in a category or measure theoretic sense. However, it contains the union over arbitrarily long words of the subshifts of finite type obtained by omitting from the two-sided $N$-shift sequences that contain the word anywhere in their length. For $\mu$ the measure of maximal entropy on such a subshift $h_{\mu}(\sigma)$ can be made as close as is desired to $\log N$ by taking the word long enough. Hence the set of non-doubly transitive points is of full Hausdorff dimension. Hausdorf dimension is a much more delicate notion of which subsets are negligible.

Of the three quantities we have been considering - positive Lyapunov exponent, Hausdorff dimension and entropy - the entropy is the most fundamental. A homeomorphism $f: M \rightarrow M$ of a compact Hausdorff space $M$ determines the Borel $\sigma$ algebra, the family of $f$-invariant regular Borel probability measures, their entropies $h_{\mu}(f)$ and the topological entropy satisfying Dinaburg's Theorem [7] that

$$
h(f)=\sup _{\mu} h_{\mu}(f) \text {. }
$$

The measure $\mu$ determines the set $G_{\mu}$ of its positive generic points. 
If $M$ is metrizable then a choice of a Lipschitz equivalence class of metrics determines the Hausdorff dimension of $G_{\mu}$. If $M$ has a differential manifold structure then choice of such a structure determines the $n$ Lyapunov exponents of $\mu$, provided that $f$ is a diffeomorphism of the structure. Here $n$ is the dimension of $M$ as a topological space. If $f$ is of class $C^{1+\alpha}$ in the chosen differential structure Pesin constructs local stable and unstable manifolds [16], [20]. If $n=2$ and a structure is chosen in which $f$ is $C^{1}$ and satisfies Smale's Axiom A then it has $C^{1}$ unstable manifolds which cut $G_{\mu}$ in the sets considered in our theorem. Choice of a differential structure determines the Lipschitz equivalence class of metrics given by the continuous Riemannian metrics on the manifold and this now determines $\delta_{\mu}$ and $\chi_{\mu}$. (Incidentally, Ledrappier has recently introduced in [13] a 'dimension' for a measure $\mu$ related to our $\delta_{\mu}$ but defined without reference to a transformation $f$.)

A diffeomorphism satisfying the hypotheses of our theorem and strong transversality is structurally stable [18], [22]. Indeed, any Anosov diffeomorphism $f$ of $T^{2}$ is topologically conjugate to a linear one, $g$ say, $[8]$. By means of carrying the differential structure across by the conjugacy $j$ we can regard $f$ as $g$ with a new differential structure. The invariant measures $\mu$ and their entropies $h_{\mu}(g)$ are unchanged but the Lyapunov exponent $\chi_{\mu}$ of $g$ may have changed and, since the new differential structure determines a different class of metrics, so too may the Hausdorff dimension $\delta_{\mu}$ even though the set $G_{\mu} \cap W^{\mu}(x, g)$ is the same. The theorem applies to show that the new values of $\chi_{\mu}$ and $\delta_{\mu}$ for $g$ still satisfy

$$
h_{\mu}(g)=\delta_{\mu} \chi_{\mu}
$$

but this is just the theorem applied to $f$ and $j_{*} \mu$.

The author gratefully acknowledges the financial support of the Stiftung Volkswagenwerk for a visit to the IHES in September 1980 during which part of this work was done.

\section{REFERENCES}

[1] R. Adler \& B. Marcus. Topological entropy and equivalence of dynamical systems. Mem. Amer. Math. Soc. 219 (1979).

[2] R. Bowen. Topological entropy for non-compact sets. Trans. Amer. Math. Soc. 184 (1973), $125-136$.

[3] R. Bowen. Equilibrium states and the ergodic theory of Anosov diffeomorphisms. Lecture Notes in Maths. No. 470. Springer: Berlin, 1975.

[4] R. Bowen. Hausdorff dimension of quasi-circles. Publ. Math. I.H.E.S. 50 (1979), 11-26.

[5] R. Bowen \& D. Ruelle. The ergodic theory of Axiom A flows. Inv. Math. 29 (1975), 181-202.

[6] M. Denker, C. Grillenberger \& K. Sigmund. Ergodic theory on compact spaces. Lecture Notes in Maths. No. 527. Springer: Berlin, 1976.

[7] E. I. Dinaburg. On the relations among various entropy characteristics of dynamical systems. Math. USSR Izv. 5 (1971), 337-378.

[8] J. Franks. Anosov diffeomorphisms. In Global Analysis, Proc. Symp. Pure Math. XIV, Amer Math. Soc., pp. 61-93. Providence R.I., 1970.

[9] H. Furstenberg. Disjointness in ergodic theory, minimal sets and a problem in Diophantine approximation. Math. Systems Theory 1 (1967), 1-49.

[10] M. Hirsch \& C. Pugh. Stable manifolds and hyperbolic sets. In Global Analysis, Proc. Symp. Pure Math. XIV, Amer. Math. Soc., pp. 133-164. Providence R.I., 1970. 
[11] W. Hurewicz \& H. Wallman. Dimension Theory. Princeton University Press: Princeton, 1941.

[12] A. Katok. Lyapunov exponents, entropy and periodic orbits for diffeomorphisms. Publ. Math. I.H.E.S. 51 (1980), 137-174.

[13] F. Ledrappier. Some relations between dimension and Lyapunov exponents. Preprint. CNRS, 1981.

[14] H. McCluskey \& A. Manning. Hausdorff dimension for horseshoes. In preparation.

[15] Z. Nitecki. Differentiable Dynamics. MIT Press: Cambridge, 1971.

[16] Ya. B. Pesin. Invariant manifold families which correspond to nonvanishing characteristic exponents. Math. USSR Izv. 10 (1976), 1261-1305.

[17] Ya. B. Pesin. Lyapunov characteristic exponents and smooth ergodic theory. Russ. Math. Surveys 32 No. 4 (1977), 55-114.

[18] C. Robinson. Structural stability of $C^{1}$ diffeomorphisms. J. Diff. Equations 22 (1976), $28-73$.

[19] D. Ruelle. An inequality for the entropy of differentiable maps. Bol. Soc. Bras. Mat. 9 (1978), 83-87.

[20] D. Ruelle. Ergodic theory of differentiable dynamical systems. Publ. Math. I.H.E.S. 50 (1979), 27-58.

[21] Ya. G. Sinai. Gibbs measures in ergodic theory. Russ. Math. Surveys 27 No. 4 (1972), 21-69.

[22] S. Smale. Differentiable dynamical systems. Bull. Amer. Math. Soc. 73 (1967), 747-817. 